\title{
Cystic lesions of the pancreas
}

\section{Ioannis Karoumpalis ${ }^{\text {, Dimitrios K. Christodoulou }}{ }^{\mathrm{b}}$}

General Hospital of Athens G. Gennimatas, Athens; University Hospital of Ioannina, Ioannina, Greece

\section{Abstract}

\begin{abstract}
Different types of benign or malignant cystic lesions can be observed in the pancreas. Pancreatic cystic lesions are classified under pathology terms into simple retention cysts, pseudocysts and cystic neoplasms. Mucinous cystic neoplasm is a frequent type of cystic neoplasm and has a malignant potential. Serous cystadenoma follows in frequency and is usually benign. Intraductal papillary mucinous neoplasms are the most commonly resected cystic pancreatic neoplasms characterized by dilated segments of the main pancreatic duct and/or side branches, the wall of which is covered by mucus secreting cells. These neoplasms can occupy the pancreatic head or any part of the organ. Solid pseudopapillary tumor is rare, has a low tendency for malignancy, and is usually located in the pancreatic body or tail. Endoscopic ultrasound with the use of fineneedle aspiration and cytology permits discrimination of those lesions. In this review, the main characteristics of those lesions are presented, as well as recommendations regarding their follow up and management according to recent guidelines.
\end{abstract}

Keywords Pancreatic cystic tumors, pancreatic cysts, serous cystadenoma, mucinous cystic neoplasm, intraductal papillary mucinous neoplasia

Ann Gastroenterol 2016; 29 (2): 155-161

\section{Introduction}

Different types of benign or malignant cystic lesions can be observed in the pancreas. Pancreatic cystic lesions are classified under pathology terms into simple retention cysts, pseudocysts and cystic neoplasms [1]. The non-neoplastic cysts are better divided histologically into epithelial cysts (with retention cysts being the most common) and non-epithelial ones (with pseudocysts being the most common) [2]. Distinguishing between the various types of lesions has important prognostic and therapeutic implications. Cystic pancreatic lesions may be associated with systemic disease such as cystic fibrosis or von Hippel-Lindau disease. Pancreatic tumors can be identified in up to $80 \%$ of patients with multiple endocrine neoplasia type 1 , as shown by intensive anatomic studies of the pancreatic tissue $[1,3,4]$.

Division of Gastroenterology, a General Hospital of Athens "G. Gennimatas", Athens (Ioannis Karoumpalis); ${ }^{\text {bUniversity Hospital }}$ of Ioannina, Ioannina (Dimitrios K. Christodoulou), Greece

\section{Conflict of Interest: None}

Correspondence to: Dimitrios Christodoulou, Ass. Professor of Gastroenterology, Department of Gastroenterology, Division of Internal Medicine, Faculty of Medicine, School of Health Sciences, University of Ioannina, 45110 Ioannina, Greece, Tel.: +30 2651007611 , Fax: +30 2651007883 , e-mail: dchristo@uoi.gr

Received 14 January 2015; accepted 26 January 2016

DOI: http://dx.doi.org/10.20524/aog.2016.0007

\section{Retention cysts}

Retention cysts, also called true or simple cysts, are usually found incidentally during an imaging study and have no clinical significance. They are usually small and their wall is covered by normal epithelium with ductal and centroacinar cells. They are observed in $25 \%$ of patients with cystic fibrosis. No treatment is necessary for these benign cysts [5].

\section{Pseudocysts}

Pseudocysts of the pancreas are a consequence of acute pancreatitis with significant inflammation and some degree of necrosis. Pseudocysts contain pancreatic fluid rich in amylase and other pancreatic enzymes and they usually communicate with the pancreatic ducts. They do not have a true wall with normal pancreatic cells, their wall is formed by fibrous and granulation tissue. The absence of true epithelium in the wall of pseudocysts is related to their nature and is one of their main characteristics. The finding of a pancreatic cystic lesion in a patient with a history of acute pancreatitis with or without amylase elevation should raise suspicion for a pseudocyst. The fluid of the cyst, if aspirated, is usually dark in color and contains pancreatic enzymes and bicarbonates. The communication of the pseudocyst with the pancreatic ductal system and its exact size and location can be demonstrated by imaging studies such as magnetic resonance cholangiopancreatography (MRCP), endoscopic ultrasonography (EUS) and endoscopic retrograde cholangiopancreatography (ERCP) if necessary [6]. 
The management of pseudocysts should be directed by symptoms. In the absence of symptoms, pseudocysts can be followed up and no treatment is necessary if they are not increasing in size. Symptomatic cysts require treatment either with drainage or resection. Endoscopic drainage under EUS guidance through the stomach or the duodenal wall is performed in advanced endoscopic centers (Fig. 1). Pseudocysts can also be drained surgically by anastomosing the cystic wall to the stomach, duodenum or jejunum as appropriate.

\section{Cystic neoplasms}

Cystic pancreatic neoplasms include serous cystadenoma, mucinous cystadenoma/cystadenocarcinoma, intraductal papillary mucinous neoplasm (IPMN) and solid pseudopapillary tumor, also named papillary cystic neoplasm or pseudopapillary neoplasm (Table 1) $[1,4]$.

In the past, mucinous cystadenoma was considered the most frequent pancreatic cystic neoplasm, followed by serous cystadenoma. Solid pseudopapillary tumor is rather rare. Pancreatic cystic neoplasms should be evaluated appropriately due to their risk of malignancy, depending on their type (Table 2) [7,8]. IPMN is the most commonly resected cystic pancreatic neoplasm. The incidence of cystic neoplasms is increasing with old age and in elderly patients, the most common cystic neoplasm being IPMN. Moreover, small incidental cysts in asymptomatic patients correspond to small branch-duct IPMN. Crippa et al reported that mucinous cystic neoplasms represent $25 \%$ of all pancreatic cystic neoplasms and IPMNs 50\% of them [9]. Another large series of 851 patients who underwent surgical resection for a cystic pancreatic neoplasm from 1978 to 2011 showed that IPMNs accounted for $38 \%$ of lesions, mucinous cystic neoplasms for $23 \%$, serous cystic tumors for $16 \%$, and solid pseudopapillary tumors for $3 \%[10]$.

EUS findings by themselves are not accurate enough to diagnose the type of cystic lesion of the pancreas definitively, or to determine its malignant potential in all cases. EUSguided fine-needle aspiration (EUS-FNA) with assessment of tumor markers and cytological examination of the cystic fluid increases the accuracy. FNA is generally safe. Prophylactic antibiotics should be administered to patients undergoing EUS-FNA, ERCP, or endoscopic drainage procedures for cystic lesions of the pancreas.

The evaluation of cystic pancreatic lesions cannot always lead to definitive conclusions about their type and benign or malignant nature. EUS-FNA of the fluid and wall of the cyst is important and permits cytology and measurement of carcinoembryonic antigen (CEA). Antibiotic prophylaxis is important when puncturing a pancreatic cyst and should be given prior to the procedure.

\section{Mucinous cystic neoplasms}

Mucinous cystic neoplasms contain septated fluid cavities of $1-2 \mathrm{~cm}$ in size (Fig. 2). They are malignant in about $25 \%$

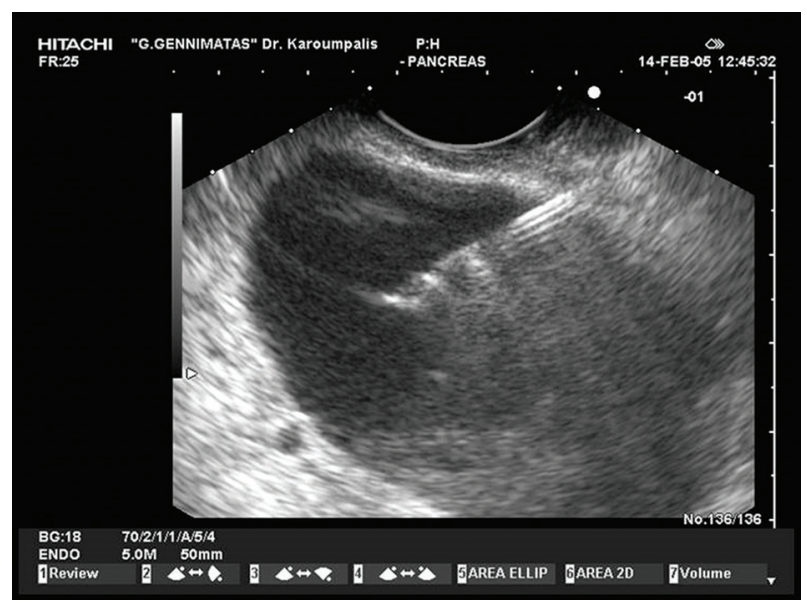

Figure $1 \mathrm{~A}$ pancreatic pseudocyst during drainage. The fine needle can be observed inside the lesion during drainage. The material of the pseudocyst appears somewhat thick and viscous

Table 1 The World Health Organization classification of pancreatic tumors [4]

\begin{tabular}{|l}
\hline Benign \\
\hline Serous cystadenoma \\
Mucinous cystadenoma \\
\hline Intraductal papillary mucinous adenoma \\
\hline Mature teratoma \\
\hline Borderline \\
\hline Mucinous cystic tumor with moderate dysplasia \\
\hline Intraductal mucinous papillary tumor with moderate dysplasia \\
\hline Solid pseudopapillary tumor \\
\hline Malignant \\
\hline Highly ductal dysplasia, carcinoma in situ \\
\hline Ductal adenocarcinoma \\
\hline Serous cystadenocarcinoma \\
\hline Mucinous cystadenocarcinoma \\
\hline Intraductal papillary mucinous carcinoma \\
\hline Acinar-cell carcinoma \\
\hline Solid pseudopapillary carcinoma \\
\hline Pancreatic blastoma \\
\hline Osteoclasts similar to giant-cell tumor \\
\hline Mixed carcinoma \\
\hline
\end{tabular}

of cases. The cystic wall is thin and may contain calcification at some parts of it in up to $15 \%$ of patients (pathognomonic feature). The cavities communicate rarely with the pancreatic ducts. Features predictive of malignancy include wall irregularity or focal thickening, large size and solid regions inside or outside the cyst $[4,11]$. Aspiration of the fluid during EUS-FNA may be difficult due to the viscous nature of the mucus and sometimes requires a larger caliber (19 gauge) needle and persistence. High levels of CEA in the cystic fluid 
Table 2 Differential diagnosis of cystic lesions of the pancreas

\begin{tabular}{lllll}
\hline Type & Age (years) & Location in pancreas & Malignant potential & Communication with the duct \\
\hline Retention cyst & & Mainly head & None & Rarely \\
Pseudocyst & & Anywhere & None & Frequently \\
Serous cystadenoma & $50-70$ & Anywhere & Very low & Rarely \\
Mucinous cystadenoma & $40-50$ & Mainly body and tail & High & Occasionally \\
Intraductal papillary mucinous neoplasm & $60-70$ & Mainly head & Moderate-high & Typically \\
Solid pseudopapillary tumor & $20-40$ & Anywhere & Moderate & Rarely \\
\hline
\end{tabular}

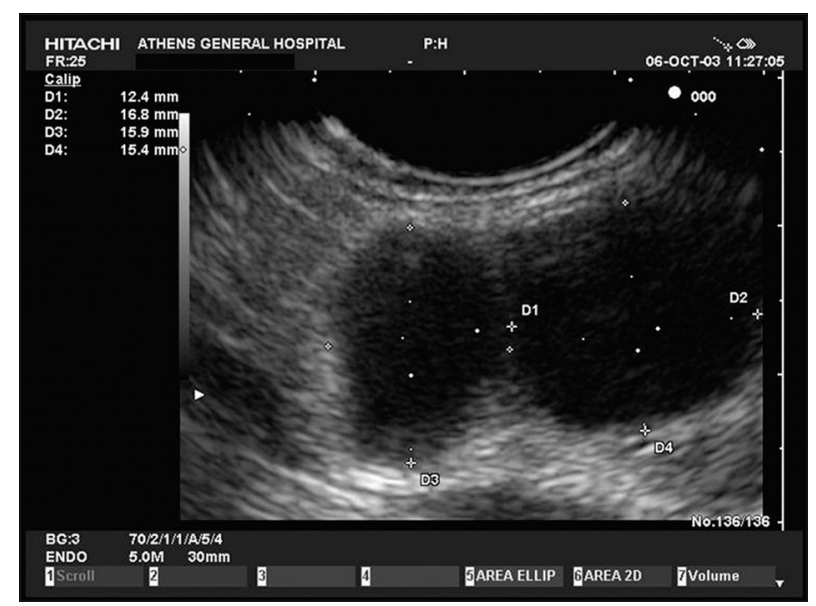

Figure 2 Pancreatic mucinous neoplasm in which the cystic cavities can be observed

and high viscosity of the fluid are compatible with a mucinous cystic lesion. Drop sign is an optical important clue, and consists of a drop of viscous mucous which is hanging from the tip of the needle and falls gradually on the side (like honey). During EUS-FNA, the endoscopist should try to obtain material from the wall of the cyst. The presence of columnar or cuboidal mucinous epithelial cells is diagnostic and is observed in up to $50 \%$ of the cases. An underlying ovarian stroma is pathognomonic and can differentiate mucinous cystadenoma from IPMN. The patchy distribution of the wall lesions can decrease the diagnostic accuracy or EUS-FNA or biopsy, so the endoscopist should select the regions of the wall with solid component if present and insist on poking the cystic wall to check for invasive component. Mucinous cystadenomas should generally be referred for surgery due to their high probability for malignant transformation. Old age and comorbidities may alter the therapeutic strategy in favor of regular follow up. Malignancy is associated with epithelial nodules in the cyst of the mucinous cystic neoplasm, but most echogenic lesions detected in cysts by EUS are mucus. Position change and EUSFNA can distinguish mucus from epithelial nodules [12].

\section{IPMNs}

IPMNs are usually found in men aged over 60 years old, frequently after one or more episodes of acute pancreatitis due to obstruction of the pancreatic duct by mucus plugs. The etiology of IPMNs is unknown and they have been associated with some other diseases such as non-pancreatic tumors, familial polyposis syndrome and Peutz-Jeghers syndrome.

IPMNs occupy part or the wholelength of the main pancreatic duct and/or side pancreatic branches, and, histologically, they are characterized by a dilated main pancreatic duct or side branches, papillary overgrowth of the ductal epithelial wall and aberrant mucin production. According to these findings, IPMNs are divided into a main duct type (MDT-IPMN), a branch duct type (BDT-IPMN, often in the uncinate process), and a mixed type (combining MDT and BDT) according to the extent of involvement of the organ (Fig. 3) [9]. Their tendency for malignancy is lower compared with mucinous cystadenomas but the MDT-IPMN has usually more aggressive nature and histology than the BDT-IPMN. Most patients with IPMN do not have invasive cancer and the progression of the lesion is generally slow. The classic signs of IPMN include: a partial or diffuse dilatation of the main pancreatic duct to $10 \mathrm{~mm}$ or more without abnormalities of the common bile duct; a patulous and mucus secreting orifice of the papilla of Vater; and small cysts 5-20 mm (BDT-IPMN) or/and mural nodules at the wall of main pancreatic duct. Significant dilatation of the main pancreatic duct to over $10 \mathrm{~mm}$, cysts larger than $20 \mathrm{~mm}$, ductal filling defects, thickened septa, solid lesion and greater cyst growth rate are all suggestive of malignancy $[13,14]$. These mucin-producing tumors share some of the morphological and clinical features with mucinous cystic neoplasms, but, unlike mucinous cystic neoplasms, they are associated with ductal ectasia and intraductal papillary growth. Papillary projections of IPMN lesions can assume 1 of 4 distinct histopathological subtypes: 1) gastric subtype, the most frequent pattern in BDT-IPMN with a low malignancy potential; 2) intestinal type, the most frequent type in MDT-IPMN with a high risk to develop invasive carcinoma; 3) pancreaticobiliary subtype, usually aggressive; and 4) oncocytic type, usually non-invasive. Invasive carcinoma developing in IPMN presents either (a) as a tubular type that mimics common ductal adenocarcinoma and has similar histology and prognosis; or (b) as the colloid type, characterized by abundant mucus between scant carcinoma cells with a better overall prognosis [3].

The differential diagnosis of cystically dilated side branches includes serous cystadenoma or mucinous cystic neoplasm [15]. Sometimes IPMN can be confused with chronic pancreatitis, but the normal appearing pancreatic parenchyma and the presence of mucus are in favor of IPMN. However, chronic 


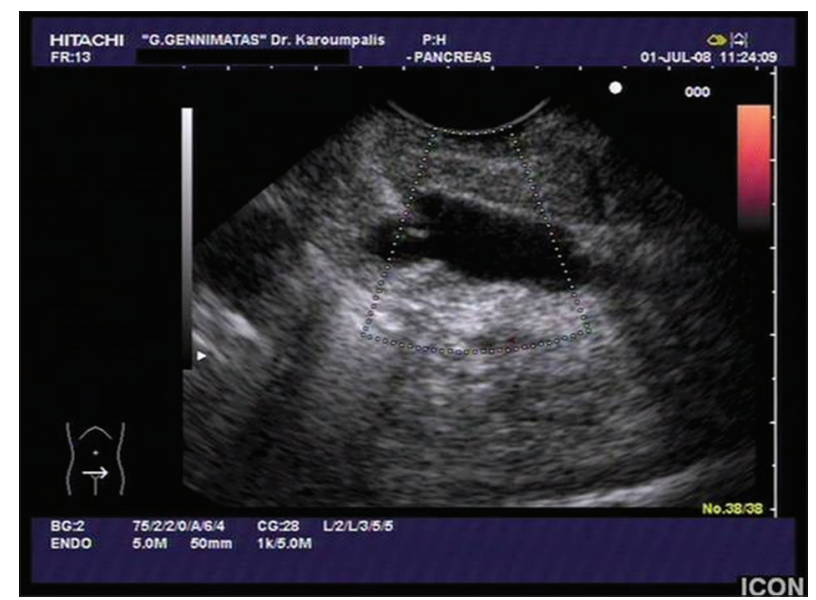

Figure 3 Intraductal papillary mucinous neoplasm. The dilatation of the main pancreatic duct can be observed

pancreatitis can complicate the course of IPMN as a result of ductal obstruction from the tumor or the mucus. Imaging features suggestive of malignant transformation include a large unilocular cystic area, focal hypoechoic areas, or mural nodules. Aggressive tumors can invade the duodenum or the common bile duct, adjacent lymph nodes, vascular structures and peripancreatic space $[16,17]$.

After the diagnosis of an IPMN, a recommendation for surgical resection is usually made if the patient is a good surgical candidate, taking into account comorbidities, patient's age and IPMN subtype [18]. In 2006, the working group of the International Association of Pancreatology proposed Consensus Guidelines for the management of BDTIPMN (Sendai Consensus Guidelines) [19]. These guidelines recommend surgical resection for all BDT-IPMN greater than $3 \mathrm{~cm}$ irrespective of symptoms, and for all BDT-IPMNs less than $3 \mathrm{~cm}$ with any one of several worrisome features including the presence of cyst-related symptoms, mural nodule, or a dilated pancreatic duct greater than $6 \mathrm{~mm}$. For presumed BDT-IPMNs less than $3 \mathrm{~cm}$, the application of the Consensus Guidelines may reduce the resection rate for lowrisk lesions [20].

\section{Serous cystadenoma}

Serous cystadenomas are benign focal cystic lesions which usually include multiple, small $(1-2 \mathrm{~cm}$ in diameter) cysts separated by thick fibrous septa, resembling a honeycomb (Fig. 4) [21]. They can be located anywhere in the pancreas and are rarely malignant, opposite to IPMNs and mucinous cysts. The presence of a central calcified or highly fibrotic area is diagnostic, but it is only observed in $10 \%$ of patients. Rarely are the cysts of serous cystadenoma larger than $2 \mathrm{~cm}$. In contrast, there is also a type with solid appearance that contains multiple small microcysts (1-2 $\mathrm{mm}$ ), is hypoechoic in imaging studies and can be misinterpreted for carcinoma. The presence of multiple cysts that contain mucin, focal duct dilatation with nodularity and thickening should direct to a mucinous tumor

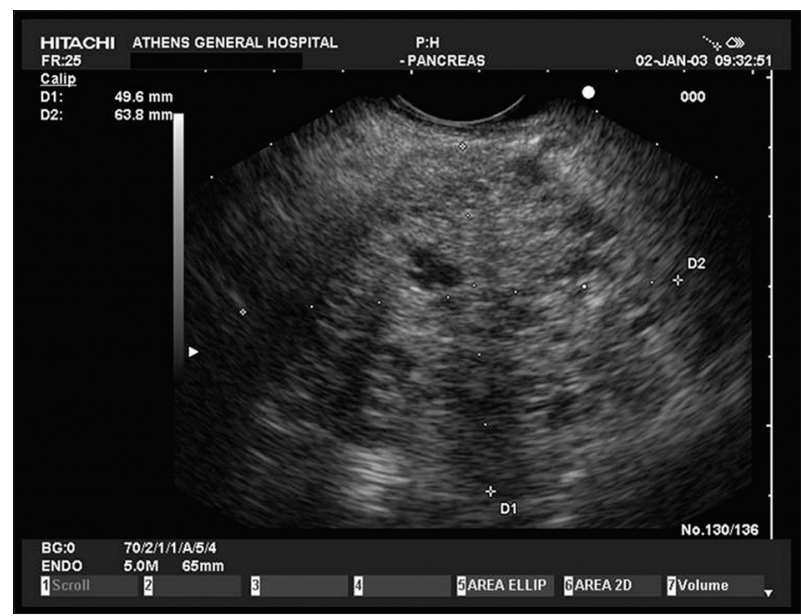

Figure 4 Serous cystadenoma. Small cystic lesions which produce a honeycomb appearance can be observed

and not a serous cystadenoma. CEA is usually within normal limits in serous cystadenomas and the fluid of the cysts has low viscosity in most cases. Cytologic evaluation after EUSFNA can establish the diagnosis in about $50 \%$ of patients, with the pathognomonic findings of bland cuboidal glycogen and staining cells. FNA carries the risk of bleeding due the increased vascularity of serous cystadenomas (with subsequent contamination of the fluid with blood) and aspiration of fluid is sometimes difficult due to the small amount of fluid in the small cysts [22].

\section{Solid pseudopapillary tumor (papillary cystic neoplasm)}

Solid pseudopapillary tumor has been mainly described in young females and is almost always located in the tail or body of pancreas. EUS reveals a tumor with mixed echogenicity (sometimes with small calcified areas) and clear margins defined by a capsule (Fig. 5). Cytopathology reveals monomorphic tumor cells with a foamy, eosinophilic cytoplasm and round nuclei. The cells form branching papillae over a fibrovascular myxoid stroma and the cystic parts of the tumor contain necrotic material and bloody dark fluid. Immunochemistry is characteristic and shows positive reactivity for alpha-1 antitrypsin, progesterone receptor, vimentin and neuron specific enolase. The compete resection of pseudopapillary tumor in clear margins is associated with excellent survival, however the tumor can recur or give metastases in some rare cases. Differential diagnosis should be made with neuroendocrine pancreatic tumors [23].

\section{Diagnostic evaluation of cystic pancreatic tumors}

There are no general guidelines for evaluation and management of pancreatic cystic lesions [24]. One should try to distinguish a pancreatic cystic tumor from a pseudocyst (10-37\% false diagnosis rate). Subsequently one should try 


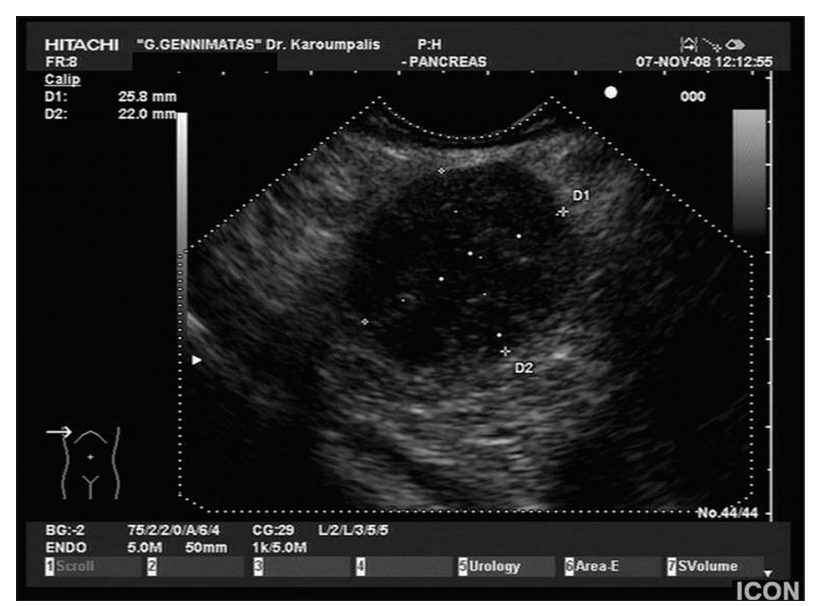

Figure 5 Solid pseudopapillary tumor. The lesions is hypoechoic and homogeneous. Differential diagnosis includes pancreatic neuroendocrine tumors

to identify the cystic tumor type and malignant potential with features such as clinical presentation, patient's age, sex, imaging findings and EUS-FNA results serving as indicators. Imaging studies, such as helical CT and magnetic resonance imaging (MRI) with MRCP offer important information. EUS is a valuable diagnostic tool for pancreatic disease and can examine not only the lesion in detail (number and size of cysts, solid components, papillary projections, number and thickness of septa, mural nodules etc.), but also the remaining pancreatic parenchyma for additional abnormalities. EUS is also performed as a preoperative tool to check for signs of malignancy and define clearly the size and margins of the lesion and potential communication with the pancreatic ductal system. Mural nodules inside the cyst, focal wall thickening, adjacent solid component and collateral vessels should raise suspicion of malignancy. Some investigators found that the presence or absence of at least 2 of three features (mural nodules, septa, parenchymal changes) offered respectively a sensitivity and specificity of $94 \%$ and $85 \%$ for the presence or not of malignancy $[8,25]$.

Recent publications, such as the Fukuoka guidelines, provide more specific recommendations for surgical resection and surveillance of mucinous cystic neoplasms and IPMNs [19]. In the study by Lee et al, a new EUS-based scoring system has been proposed for the prediction of malignancy in patients with BDT-IPMN [26]. It has been proposed that for patients $<65$ years old a threshold of $2 \mathrm{~cm}$ can be used to determine more aggressive management, while for older patients, a lesion $>3 \mathrm{~cm}$ without the presence of mural nodules could be observed. The American Gastroenterological Association published a technical review on the diagnosis and management of asymptomatic neoplastic pancreatic cysts [27]. The review concluded that for patients with benign-appearing lesions and low-risk features on imaging, surveillance with MRI and EUS with or without FNA will allow watchful waiting of a presumed mucinous lesion, including both mucinous cystic neoplasms and branch duct IPMNs. The risks of malignancy should always be weighed against the risks of pancreatic surgery.
Most importantly, the American Gastroenterological Association published a Guideline on the diagnosis and management of asymptomatic pancreatic cysts, based on expert opinions and the available literature [28]. According to their recommendations, pancreatic cysts $<3 \mathrm{~cm}$ without a solid component or dilated pancreatic ducts should be reevaluated by MRI in 1 year and every 2 years thereafter for a total of 5 years if there is no change in size and other features. In contrast, cysts with 2 or more high-risk characteristics, such as dilated main pancreatic duct, size more than $3 \mathrm{~cm}$ and the presence of a solid component, should undergo EUS-FNA. Those patients with a dilated pancreatic duct and a solid component and/or suspicious findings on EUS and EUS-FNA should undergo surgical resection of the cystic lesion to reduce mortality from malignancy.

However, some experts questioned some of the statements of the guideline, emphasized the role of EUS-FNA and concluded that we need more evidence $[29,30]$. In Fig. 6, a simplified algorithm for the follow up and treatment of pancreatic cystic neoplasms is proposed. The technique of EUS-FNA does not differ for solid or cystic lesions. For cystic lesions, broadspectrum antibiotic prophylaxis is recommended prior to the puncture procedure and should be continued for at least $48 \mathrm{~h}$. One should try to aspirate all the cystic fluid with one pass of the needle to reduce complications. Molecular analysis of cyst fluid aspiration is being investigated as a diagnostic tool for the risk assessment of cystic pancreatic neoplasms, especially for the smaller cysts which do not meet size criteria for resection [2].

The sensitivity of cytology varies depending on the expertise of the endoscopist and the cytologist. Sometimes, cytology specimens are false negative because of sampling error. Cytology should be completed with measurements of CEA, amylase levels and mucin stain to increase the diagnostic accuracy to up to $80-90 \%$ [31]. CEA measurement in the fluid is particularly helpful to separate serous from mucinous lesions, but there may be some degree of overlap. A CEA level $<5 \mathrm{ng} / \mathrm{mL}$ is suggestive of serous cystadenoma, while values $>400 \mathrm{ng} / \mathrm{mL}$ provide almost $100 \%$ specificity in diagnosing mucinous cystic neoplasms from pseudocysts. Despite this, infected pseudocysts may sometimes have elevated fluid CEA levels. High cystic fluid amylase levels are observed in cysts that communicate with the pancreatic ducts such as pseudocysts and IPMNs [32]. All these pieces of information combined can usually lead to the correct diagnosis.

Cystic fluid from EUS-FNA is prioritized for CEA, amylase, mucin stain, and cytologic analysis. FNA is important even for classically benign appearing lesions to rule out malignant potential. FNA is not advised for probable malignant lesions for which surgery is already scheduled. However, the finding of a malignant or mucinous cytology, and an elevated fluid CEA may sometimes encourage decision for resection.

\section{Concluding remarks}

Pancreatic cystic lesions are infrequent but can pose a diagnostic challenge. They can represent simple cysts, 


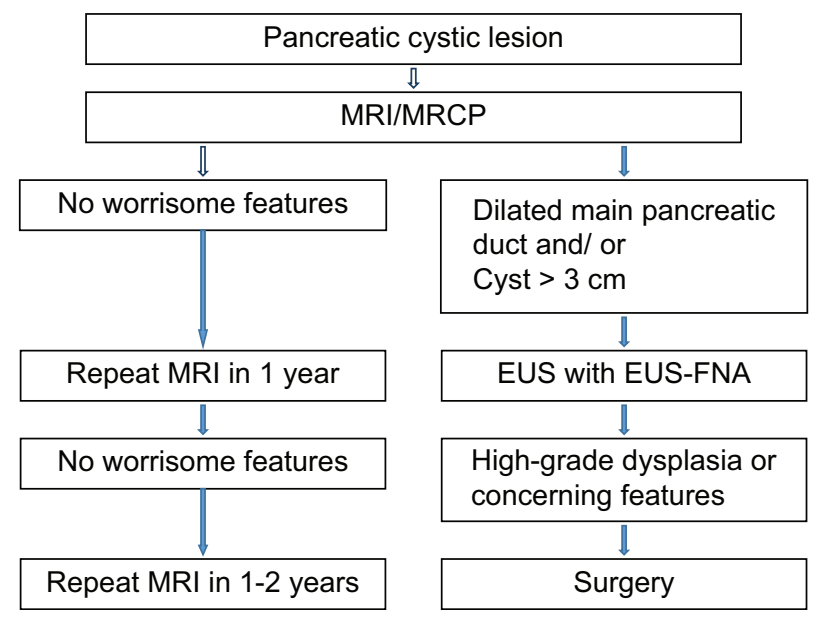

Figure 6 Simple algorithm for the follow up and treatment of pancreatic cysticlesions in accordance with recent AGA guidelines (simplified) [28] $M R I$, magnetic resonance imaging; MRCP, magnetic resonance cholangiopancreatography; EUS, endoscopic ultrasound; FNA, fine-needle aspiration

pseudocysts due to previous acute or chronic pancreatitis or cystic pancreatic tumors. MRI is an important diagnostic tool for the evaluation of pancreatic cystic lesions and it should be followed by EUS with EUS-FNA where worrisome features are recognized. In doubtful cases it is better to proceed with EUS. Cystic pancreatic neoplasms are increasingly recognized and information provided by EUS with cytology and fluid examination can offer important assistance in the evaluation and management. However, the results of EUS should be combined with the clinical history, laboratory findings and other imaging studies (such as CT and MRI/MRCP) to promote the early detection of the cystic lesion, define the type of the tumor and distinguish it from pseudocysts, in order to recommend the most appropriate treatment [4].

\section{References}

1. Dietrich CF, Jenssen C. Benign and malignant cystic tumors of the pancreas. In: Dietrich CF, editor. Endoscopic ultrasound - an introductory manual and atlas. $1^{\text {st }}$ ed. New York: Thieme; 2006:208-229.

2. Garud SS, Willingahm FF. Molecular analysis of cyst fluid aspiration in the diagnosis and risk assessment of cystic lesions of the pancreas. Clin Transl Sci 2012;5:102-107.

3. Al-Haddad M, Schmidt MC, Sandrasegaran K, Dewitt J. Diagnosis and treatment of cystic pancreatic tumors. Clin Gastroenterol Hepatol 2011;9:635-648.

4. Levy MJ. Pancreatic cysts. Gastrointest Endosc 2009;69:S110-S116.

5. Dietrich CF, Chichakli M, Hirche TO, et al. Sonographic findings of the hepatobiliary-pancreatic system in adult patients with cystic fibrosis. J Ultrasound Med 2002;21:409-416.

6. Giovannini M, Pesenti C, Rolland AL, Moutardier V, Delpero JR. Endoscopic ultrasound-guided drainage of pancreatic pseudocysts or pancreatic abscesses using a therapeutic echo endoscope. Endoscopy 2001;33:473-477.
7. Frossard JL, Amouyal P, Amouyal G, et al. Performance of endosonography-guided fine needle aspiration and biopsy in the diagnosis of pancreatic cystic lesions. Am J Gastroenterol 2003;98:1516-1524.

8. Brugge WR, Lewandrowski K, Lee-Lewandrowksi E, et al. Diagnosis of pancreatic cystic neoplasms: a report of the Cooperative Pancreatic Cyst Study. Gastroenterology 2004;126:1330-1336.

9. Crippa S, Fernandez-del-Castillo C, Salvia R, et al. Mucinproducing neoplasms of the pancreas: an analysis of distinguishing clinical and epidemiological characteristis. Clin Gastroenterol Hepatol 2010;8:213-219.

10. Valsangar NP, Morales-Oyarvide V, Thayer SP, et al. 851 resected cystic tumors of the pancreas: a 33-year experience at the Massachussets General Hospital. Surgery 2012;152:S4-S12.

11. Gress F, Gottlieb K, Cummings O, et al. Endoscopic ultrasound characteristics of mucinous cystic neoplasms of the pancreas. Am J Gastroenterol 2000;95:961-965.

12. Zhong N, Zhang L, Takahashi N, et al. Histologic and imaging features of mural nodules in mucinous pancreatic cysts. Clin Gastroenterol Hepatol 2012;10:192-198.

13. Kang MJ, Jang JY, Kim SJ, et al. Cyst growth rate predicts malignancy in patients with branch duct intraductal papillary mucinous neoplasms. Clin Gastroenterol Hepatol 2011;9:87-93.

14. Pais SA, Attasaranya S, Leblanc JK, Sherman S, Schmidt CM, Dewitt J. Role of endoscopic ultrasound in the diagnosis of intraductal papillary mucinous neoplasms: correlation with surgical histopathology. Clin Gastroenterol Hepatol 2007;5:489-495.

15. Brugge WR. Evaluation of pancreatic cystic lesions with EUS. Gastrointest Endosc 2004;59:698-707.

16. Cellier C, Cuillerier E, Palazzo L, et al. Intraductal papillary and mucinous tumours of the pancreas: accuracy of preoperative computed tomography, endoscopic retrograde pancreatography and endoscopic ultrasonography, and long-term outcome in a large surgical series. Gastrointest Endosc 1998;47:42-49.

17. Seo DW, Kang GH. Twenty-six cases of mucinous ductal ectasia of the pancreas. Gastrointest Endosc 1999;50:592-594.

18. Sahani DV, Lin DJ, Venkatesan AM, et al. Multidisciplinary approach to diagnosis and management of intraductal papillary mucinous neoplasms of the pancreas. Clin Gastroenterol Hepatol 2009;7:259-269.

19. Tanaka M, Chari S, Adsay V, et al. International consensus guidelines for management of intraductal papillary mucinous neoplasms and mucinous cystic neoplasms of the pancreas. Pancreatology 2006;6:17-32.

20. Tang RS, Weinberg B, Dawson DW, et al. Evaluation of the guidelines for management of pancreatic branch-duct intraductal papillary mucinous neoplasm. Clin Gastroenterol Hepatol 2008;6:815-819.

21. Wargo JA, Fernandez-del-Castillo C, Warshaw AL. Management of pancreatic serous cystadenomas. Adv Surg 2009;43:23-34.

22. Tseng JF, Warshaw AL, Sahani DV, et al. Serous cystadenoma of the pancreas: tumor growth rates and recommendations for treatment. Ann Surg 2005;242:413-421.

23. Master SS, Savides TJ. Diagnosis of solid-pseudopapillary neoplasm of the pancreas by EUS-guided FNA. Gastrointest Endosc 2003;57:965-968.

24. Buscaglia JM, Shin EJ, Giday SA, et al. Awareness of guidelines and trends in the management of suspected pancreatic cystic neoplasms: survey results among general gastroenterologists and EUS specialists. Gastrointest Endosc 2009;69:813-820.

25. Hernandez LV, Mishra G, Forsmark C, et al. Role of endoscopic ultrasound (EUS) adn EUS-guided fine needle aspiration in the diagnosis of treatment of cystic lesions of the pancreas. Pancreas 2002;25:222-228.

26. Lee KH, Lee SJ, Lee JK, et al. Prediction of malignancy with 
endoscopic ultrasonography in patients with branch ducttype intraductal papillary mucinous neoplasm. Pancreas 2014;43:1306-1311.

27. Scheiman JM, Hwang JH, Moayyedi P. American Gastroenterological Association Technical Review on the diagnosis and management of asymptomatic neoplastic pancreatic cysts. Gastroenterology 2015;148:824-848.

28. Vege SS, Ziring B, Jain R, Moayyedi P. American Gastroenterological Association Institute Guideline on the diagnosis and management of asymptomatic neoplastic pancreatic cysts. Gastroenterology 2015;148:819-822.
29. Fernandez-del-Castillo C, Tanaka M. Management of pancreatic cysts: the evidence is not here yet. Gastroenterology 2015;148:685-687.

30. Canto MI, Hruban RH. Managing pancreatic cysts: less is more? Gastroenterology 2015;148:688-691.

31. Sand JA, Hyoty MK, Mattila J, et al. Clinical assessment compared with cyst fluid analysis in the differential diagnosis of cystic lesions in teh pancreas. Surgery 1996;119:275-280.

32. Hammel P, Levy P, Voitot $\mathrm{H}$, et al. Preoperative cyst fluid analysis is useful for the differential diagnosis of cystic lesions of the pancreas. Gastroenterology 1995;108:1230-1235. 